\title{
The role of communication in the management of tuberculosis disease
}

\author{
Beatrice Achieng Koech, Dr. Erneo Nyakundi Nyamboga \\ (Department of Communication Studies, Moi University, Kenya)
}

\begin{abstract}
Communication is about accessing information which can lead to behavioural change. The kind of communication can be between the healthcare worker and the tuberculosis patient or it can also be between the healthcare worker and the tuberculosis kin. This communication can lead to information sharing which can influence the health-seeking behavior of the TB patient. The kind of messages received by the patient apart from influencing behavior change they can also encourage public discourse on TB leading to self disclosure..This paper discusses the role of communication in the management of tuberculosis disease.
\end{abstract}

Keywords- Behaviour change, Communication, health-seeking behaviour, Public discourse, Self-disclosure

\section{INTRODUCTION}

Communication is a vital element in any development activity. Communication apart from preparing people for change, it provides information on key areas and helps in decision-making. The idea of getting individuals to adopt new kinds of behaviour has been the chief aim of the health sector for a number of decades. Good communication is the pillar for building a sense of cooperation with health care providers, beneficiaries and other stakeholders. This communication may range from something as simple as ensuring awareness regarding the disease, symptoms and treatment, to promoting the successful exchange of valuable technical information, through to the simple act of putting up messages in outdoor clinics (Agarwal \& Chauhan, 2005).Communication expands both knowledge of and access to quality services and the products needed to practice health behaviours. Communication creates demand and helps patients understand the benefits of new behaviours. It can strengthen social networks and reflect norms that support healthy behaviour. Communication also helps providers explain services and treatments to patients and encourage them to use those services consistently and effectively (Storey et al., 2014). Communication is important not only in the context of doctor-patient or family member's interaction, but also between healthcare professionals. The advantages to the patient are obvious as effective communication gives them better insight into their conditions and may aid compliance and quality of life. Poor communication with patients is thought to contribute to psychological morbidity, low personal achievement and emotional burnout (Khan, 2006). Raviglione (2010) argues that communication is concerned with informing and enhancing knowledge among the general public and people with TB and also empowering them to express their needs and take action.

\section{COMMUNICATION IN THE MANAGEMENT OF TUBERCULOSIS}

Communication should be placed centrally on the tuberculosis (TB) agenda. Many logistical and medical components of the global response to TB are relatively robust yet the communication part is not. Cure rates are high, but the numbers of people with TB who require medical help are low. Better communication, which includes advocacy, social mobilization and program communication would provide much of the solution to this and a number of other problems within today's fight against TB (Sarkar \& Scalway, 2005). All communication activities make use of some form of media or channel of communication for example mass media, community media or interpersonal communication. Communication practitioners stress that for communication to be effective, it should be comprehendible as a two-way process with 'participation' and 'dialogue' as key elements (Deane \& Parks, 2006).

\section{TUBERCULOSIS AWARENESS}

The aim of communication is to increase awareness of the community regarding basic information about tuberculosis (Communication disease health education and training (HEAT) module, 2015). The module indicates that the availability of abundant information about this disease and raising levels of community awareness can enable one to influence what is socially normal and acceptable. The module further indicates that this has an impact on TB control and that it also changes behaviour in both individuals and groups of people.

The National Communication Strategy for fighting Tuberculosis in Kenya report (2006) states that for communication to be effective, it must occur during all the three stages at which an individual must act on his or 
her own behalf, that is, at the Pre-Diagnosis stage followed by the Post-Diagnosis stage and lastly at the Treatment stage. The report claims that wrong decisions at any of these stages can be fatal and that each phase carries its own communication requirements, audiences and risk profile. Communication in TB management recognizes that just telling people what they should do does not necessarily mean that people will follow the prescribed message. The repeating of some information may also not encourage people to change their behaviour. People from the onset should recognize the need to access services as soon as they develop a cough which persists for more than three weeks. The confidence in the health care system, that is (interpersonal communication between providers and patients) and also having information and confidence that good quality diagnosis and treatment facilities are available within the environs is very important for the patient (Agarwal and Chauhan, 2005).

Communication is seen as having an important role in improving treatment adherence amongst $\mathrm{TB}$ patients. The aim towards a target goal of eighty five percent treatment success has been much more marked than that against case detection, although every effort must be made to maintain cure rates in many countries. Communication and social mobilization programs ensuring patient education, combined with broader community support and empowerment initiatives, are essential if cure rates are to improve and be sustained (Deane \& Parks, 2006). If existing baselines are established and the setting of specific measurable goals for achieving a critical level of awareness about TB among the population, is likely to go a long way towards developing communication interventions. The media in this case is seen to play a key role in this situation, going beyond newsroom reporting that draws on dry statistics and official press releases. TB communication must go beyond the usual flurry of media and communication around World TB Day (Sarkal \& Scalway, 2005).

\section{HEALTH-SEEKING BEHAVIOUR AMONG TUBERCULOSIS PATIENTS}

The health seeking behaviour of persons is influenced by their health and disease knowledge, beliefs, attitudes and perceptions. The reasons mentioned on behaviour can influence prevention behaviours, the timing of diagnosis and treatment, patient understanding of the diagnosis and treatment initiation, treatment adherence and interactions with health care providers. The knowledge that people have or perceive about TB even when they are not yet affected is an important determinant of their future health seeking behaviour (Auer et al., as cited by Yamira 2009). The International Standards for Tuberculosis Care (2006), indicates that quality of care accorded to patients and especially to TB patients determines the health-seeking behaviour of these patients. Cultural beliefs and religion were also found to influence health-seeking behaviour of patients. The understanding of traditionally held beliefs in causes and characteristics of TB is important for facilitating communication about the disease to the population and a good knowledge level among patients will positively influence treatment delay and also help reduce TB related stigma (Annan et al., 2013). Wang et al., (2008), as cited by Abebe et al., (2010) in a study reported that males were more likely to know the cause of TB compared with females. It was reported that the poor knowledge among women and non-educated individuals concerning the cause of TB will result in inappropriate health care seeking behaviour. In the study conducted by Abebe et al., (2010) it was reported that health care seeking behaviour of the study participants was poor. The majority of them did not seek help for their illness as a result of wrong perceptions and lack of financial resources mainly for transport. A significant number of them did nothing since they considered that their illness was not severe.

Raising awareness and educating community members about TB, is key for changing behaviour. The publication also claims that stigma is often a problem, especially in countries severely affected by HIV. The publication emphasizes that effective communication starts with personal communication between health workers and the people with TB, their families and local communities and this help build a supportive environment for people who feel ill and may have TB (WHO, 2008). On the other hand Sarkal and Scalway (2005), argue that there are very few countries that have conducted surveys to assess awareness and knowledge levels about TB among their populations. The little that is known, either through surveys or anecdotal evidence, points to a fairly low level of public awareness-diagnostic or treatment services available. Lack of adequate information is one of the reasons that affect health-seeking behaviour of TB patients. The report explicates how TB patients in Kenya should be receiving basic information about TB from health facility providers. The report also claims that a large number of TB patients obtained information on TB signs and symptoms and were also educated on how TB is spread. Despite all the information obtained from the providers, there were still gaps in the provider's communication regarding TB treatment, screening of close contacts and counselling patients on HIV (The Kenya TB Care II report, 2013).Health promotion programs worldwide have long been premised on the idea that providing knowledge about causes of ill health and choices available will go a long way towards promoting a change in individual behaviour. There is already a growing recognition, in both developed and developing countries, that providing education and knowledge at the individual level is not sufficient in itself to promote a change in behaviour. Health seeking behaviour clearly varies for the same individuals or communities when faced with different illnesses. Several studies of health seeking behaviour in relation to TB repeatedly demonstrate that patients do not always choose a public health care facility they delay diagnosis and often do 
not complete the lengthy course of treatment necessary. Health seeking behaviour is not just a one off isolated event; it is part and parcel of a person's family or a community's identity, which is the result of an evolving mix of social, personal, cultural and experiential factors (Mackian, 2003).

\section{TUBERCULOSIS STIGMA}

A lack of understanding about Tuberculosis has led to the disease being met with high levels of stigma and discrimination. It has also resulted in patients who are diagnosed with TB to be sent away from their communities. Patients in this situation, apart from being diagnosed early enough, continue spreading the disease when they delay seeking care for fear of stigma and discrimination. Treatment default is seen as being one of the biggest problems in the treatment of tuberculosis today. This is due to the long treatment regime which requires a patient to seek treatment several times a week for at least a period not longer than six months. Despite this issue of treatment default, there is a bit of ignorance on the possible repercussions of treatment default and is common among TB patients in the developing countries. This situation is likely to make the issue worse which is reported to be the largest driver of drug resistance (Friends for International Tuberculosis Relief report, 2015).

The stigma that accompanies tuberculosis can have a detrimental effect on the individual and family and may result in their withdrawal from society because of shame and fear. There appears to be widespread ignorance which requires education of both the individual, kin and the community. The aim of this education is to provide practical strategies to help people cope with tuberculosis. Confidentiality should be given priority during the provision of these management strategies. There are many beliefs about disease are culturally sensitive, therefore health education must also be culturally sensitive and adapted to the focus on changing misconceptions about its cause and transmission, which could in turn reduce stigmatization (Tripathy, 2015).

TB stigma results in part from misinformation. Misinformation about what causes TB, how it is spread and whether it can be cured is linked to the stigmatization of this disease and of people with TB. The lack of knowledge or information has been greatly blamed for making people to be physically isolated, discriminated against and dismissed from their work places. Public education and awareness-raising programs designed to counteract myths and to encourage greater inclusion of people with TB are an essential element of any effort to combat stigma associated with TB. The core knowledge that people need to have to change their behaviour is mainly knowledge on TB symptoms and how it is spread. Equally important is the knowledge on TB's curability and the knowledge that TB treatment is offered free of charge. People also need to have knowledge that potential TB cases should rigorously seek professional care and that active TB cases should adhere to a treatment regime (Deane \& Parks, 2006).

\section{TUBERCULOSIS KNOWLEDGE}

The lack of knowledge about having a cough for more than two weeks is a possible symptom of a fatal but curable disease which prevents millions of people from seeking treatment. Studies have repeatedly showed that if people have knowledge they are far more likely to seek treatment as opposed to when they do not have any knowledge. In terms of traditional communication, the educating of people with the knowledge and persuading the people to seek treatment for TB ends up bringing challenges but is considered by many communication organizations to be a relatively straight forward process (Deane \& Parks, 2006). Several studies have been done on tuberculosis knowledge. A survey cited in 'A Media Guide' (2012) was conducted by the International Medical Foundation and Target Tuberculosis in 2011 in Lira, Makinye in Kampala and in Wobulenzi in the central region, indicated that only $24 \%$ of the people interviewed were able to mention on the correct cause of TB. The survey also found out that while a significant percentage of the interviewees were able to mention the commonest mode of transmission of pulmonary $\mathrm{TB}$, an equally significant number named sharing of plates and food, which means there is still a gap of knowledge and understanding of issues related to TB. A similar study conducted on TB knowledge by the community in south western Ethiopia, by Bati et al., (2013), revealed a low level of knowledge about the causative agent as well as about the main symptom of TB in the present study communities. The study also showed low level of overall knowledge, unfavourable attitude and poor practices towards TB especially in participants from one community. The study recommended a need for public health education on the cause, symptoms and mode of transmission of TB would be important towards the prevention.

\section{SELF-DISCLOSURE AMONG TB PATIENTS}

Zolowere et al., (2008) in study conducted in rural southern Malawi which sought to explore the relationship of persons to whom TB patients disclose their diagnosis and secondly to identify the motivations for disclosures. The study found out that self-disclosure of diagnosis to others within the patients' social environment may be problematic because the diagnosis of TB may warrant unnecessary stigma largely derived from the association of this disease with HIV infection. The study also found out that the majority of patients reported having disclosed their status to their children. The most common way of disclosure was through 
personal discussion between the patient and their significant others. In the study participants perceived that disclosure brought returns in terms of encouragement and empowerment. During the study it was found that some patients felt stigmatized or feared stigmatization following disclosure of their disease status and some patients on anti retroviral therapy for HIV felt stigmatized by fellow patients. The findings of this study suggest that TB patients in southern Malawi were interested in disclosing their TB diagnosis if they felt they would not be stigmatized or stood to gain as a result of self-disclosure. Disclosure of diagnosis was facilitated by trust, a feeling of safety and a sense of obligation to others. The perceived stigmatization of patients by other patients is a cause for concern. A similar study on the analysis and implications for addressing TB related discrimination in Nepal found out that there is a mismatch between TB patients' fears of negative responses by friends to a disclosure of TB and the reality of the encouragement and support provided by friends towards patients who actually disclosed their infection. This mismatch provides a potential avenue for interventions to reduce discrimination associated with TB. The findings state that if more patients are made aware of the support that is being made available to other TB patients by friends and neighbours, they agree to open up about their disease, with two potential benefits. The benefits can come in the form of psychological support. The patients may gain from not having to keep their illness secret and materially, the patients may gain encouragement and support themselves (Baral, 2007).

The strategy being suggested has to first confirm the cause-effect mechanism involved. If the disclosure causes supportive behaviour from others, interventions to encourage disclosure may be useful; but if the mechanism is actually that having supportive friends and neighbours facilitates disclosure, then such interventions may prove harmful. Even if the former is the case, it would be wise to test such a strategy on a small scale before expansion, to ensure that unexpected consequences do not arise. This study concluded that patients' preference to hide their TB from other members of the community seems justified, since several community members reported that they did discriminate against people with TB. Rajeshwari et al., (2005) as cited by Dhingra and Khan (2009) argue that the consequences of stigma can be seen affecting the health seeking behaviours, as patients have been known to hesitate or choose not to disclose their TB status to family or friends out of fear of being socially avoided. Stigma is a potential barrier to treatment because it makes patients reluctant to attend treatment in clinics located in their neighbourhoods and leads to non-disclosure of illness. Disclosure can play a positive role because it can help the patient mobilize support thereby facilitating adherence, and is also important for public health reasons such as avoidance of further infection transmission. Health professionals should be aware of the negative impacts of self-disclosure in social environments where disease related stigma is widespread. If a scenario occurs where consequences of disclosure are not known, then it may require a patient not to disclose or to have very selective disclosure (Gebremariam et al., 2010).

\section{PUBLIC DISCOURSE ON TUBERCULOSIS AND HOW} IT AFFECTS PATIENTS

A study by Konradsen et al., (2014), explored how patients undergoing the medical examination for TB had to have a public diagnosis. These patients were suffering from an infectious, contagious and potentially lifethreatening disease which put undue pressure on them to contact and provide the names of everyone with whom they had interacted with. The patients did not have a choice of keeping their diagnosis private towards family, friends or healthcare professionals. They had to disclose their status to everyone in public and explain to them about the disease and its treatment protocol. This involved not only people with whom they had had a close relationship but also people with whom they had only had a very brief contact. At the end of it all, these same people would disclose this information to even more people. There was a constant demand for awareness and openness during the treatment period. This kind of public discourse eventually led to changes in the social interactions of these patients which became a huge concern to them.

\section{CONCLUSION}

The successful management of tuberculosis disease can only be realized if communication strategies on the disease are improved. Public awareness campaigns also need to be intensified so that more people are able to access correct information on TB. Proper communication channels need to be enforced so that tuberculosis information can reach a majority of the population.

\section{REFERENCES}

[1] Agarwal, G., \& Chauhan, L. (2005). Tuberculosis control in India. New Delhi: Elsevier.

[2] Storey, D.,Ahanda, S. Andaluz., K, Tsoi, A., \& Matsuki, B. (2014). What is health communication and how does it affect the HIV/AIDS continuum of care? A brief primer and case study from New York. Journal of acquired immune deficiency syndrome. 66-p s 241-s249 doi: 10.1097QA1.0000000000000243 supplement article. 
[3] Khan, I. (2006). MRCP PACES Ethics and communication skills. United Kingdom: Radcliffe publishing Ltd.

[4] Raviglione, M. (2010). Tuberculosis: The essentials. (4 ${ }^{\text {th }}$ Ed.). Florida: CRC press.

[5] Sarkar, S., \& Scalway, T. (2005). Time for action on tuberculosis communication. London: The Panos Institute.

[6] Deane, J., \& Parks, W. (2006). Advocacy, communication and social mobilization to fight TB: A ten year framework for action. France: WHO Press.

[7] Communication disease health education and training (HEAT) module (2006). Retrieved from http://www.open,edu

[8] Lights of hope: A national communication strategy for fighting Tuberculosis in Kenya. Retrieved from http://www.path.org/CP_kenya_LOH

[9] Yamira, A. (2009). The ethno-medical context of tuberculosis in a northern Ecuadorian province. ProQuest.

[10] The international standards for tuberculosis care (2006). Retrieved from http://www.who.int

[11] Annan, A., Singh, J., Dogbe, J. Asante, D., \& Owusu-Dabo, E. (2013). Health-seeking behavior of tuberculosis patients and related factors in the central region of Ghana. A journal of science and technology, 33(3), 27-38.

[12] Abebe, G., Deribew, A., Apers, L. \& Woldemicheal, K. (2010). Knowledge, health-seeking behavior and perceived stigma towards tuberculosis suspects in a rural community in south-west Ethiopia. PLoS ONE 5(10): e13339 doi: 10.1371/journal.pone.0013339.

[13] World Health Organization. (2008). Retrieved on 25 ${ }^{\text {th }}$ January 2015 from http://www.who.int/

[14] The Kenya TB care II report (2013). Retrieved from http://www.who.int

[15] Mackian, S. (2003). A review of health-seeking behavior: Problems and prospects. Health systems development program. University of Manchester. HSD/WP/05/03 Manchester.

[16] Friends for international tuberculosis relief report. (2015). Retrieved from http://www.tbhelp.org/?page_id=872

[17] Tripathy, S. (2015). Tuberculosis: Manual for obstetricians and gynecologists. India: Jaypee brothers medical publishers $(\mathrm{P})$ ltd

[18] Bati, J., Mengistu, L., \& Medhin, G. (2013). Community's knowledge, attitudes and practices about tuberculosis in Itang special district, Gambella region, south western Ethiopia. BMC Public Health 2013, 13:734 Doi: 10.1186/1471-2458-13-734.

[19] Zolowere, D., Manda, K., Panulo, B., \& Muula, A. (2008). Experiences of self-disclosure among tuberculosis patients in rural southern Malawi. Rural remote health, oct-dec: 8(4): 1037, Epub.

[20] Baral, S., Karki, D., \& Newll, N., (2007). Causes of stigma and discrimination associated with tuberculosis in Nepal: A qualitative study. BMC Public Health 2007, 7:11.

[21] Dhingra, V., \& Khan, S. (2009). A sociological study on stigma among tuberculosis patients in Delhi. Indian journal of tuberculosis. Indian J Tuberc 2010; 57: 12-18.

[22] Gebremariam, M. Gunner, B., \& Frich, J. (2010). Barriers and facilitators of adherence to tuberculosis treatment in patients on concomitant tuberculosis and HIV treatment: A qualitative study. BMC Public Health 2010, 10:651 doi: 10.1186/1471-24-10-651.

[23] Konradsen, H., Lilleback, T., \& Lomberg, K. (2014). Being publicly diagnosed: A grounded theory study of Danish patients with tuberculosis. Doi:10.3402/gh10.v9.23644 